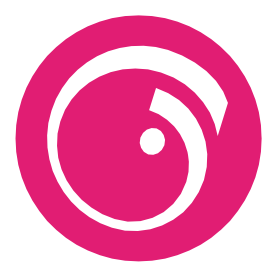

\title{
Archives Of Breast

\section{Spindle Cell Carcinoma of the Breast: A Case Report Representing Diagnostics, Therapeutics, Intervention, and Outcome}

\author{
Mohammad Naser Athamnah ${ }^{a}$, Shatha M. Al-Barrak ${ }^{b}$, Nimah A. Rabai ${ }^{a}$, Tea Natelauric, Hussein S O Al \\ Azzam $^{\mathrm{a}}$, Mohammed Alorjani ${ }^{\mathrm{d}}$, Omar M. Al-Rusan ${ }^{\mathrm{d}}$, Amer Abu-Shanab*e $^{\star \mathrm{e}}$ \\ aDepartment of General Surgery, Princess Basma Teaching Hospital, Irbid, Jordan \\ ${ }^{b}$ Department of Acute Medicine, Ninewells Hospital, NHS Tayside, Scotland \\ ${ }^{c}$ Clinical Oncology Department, EVEX Hospitals, Georgia. \\ ${ }^{d}$ Department of Pathology and Microbiology, Faculty of Medicine, Jordan University of Science and Technology, Irbid, Jordan \\ eOffice of Scientific Affairs and Research, King Hussein Cancer Center, Amman, Jordan
}

ARTICLE INFO

Received:

31 May 2021

Revised:

02 July 2021

Accepted:

07 July 2021

\section{ABSTRACT}

Background: Spindle cell carcinoma (SpCC) is an unusual form of squamous cell carcinoma (SCC) and can sometimes present in the breast. Owing to the rarity of breast SpCC, few case studies are available nowadays and proper evidence is scarce.

Case presentation: We herein report a 60-year-old female patient, who was referred to the surgery services after presenting with a right breast ulcerated mass. On physical examination, a 7x7cm mass was found along with a $3 \mathrm{~cm}$ ulcer on the top of it. Both mammography and ultrasound showed a dense mass, and tru-cut and skin punch biopsies confirmed neoplastic spindle cells within the lesion. The patient underwent a right total mastectomy with sentinel lymph node biopsies with no further chemotherapy or radiotherapy.

Conclusion: Owing to the heterogeneity of SpCC, there is no exact treatment protocol for this type of cancer, and mastectomy or conservative surgery can be performed in certain groups of patients depending on tumor size, stage, and lymph node involvement. Fortunately, promising medical and biological therapies might be of use in the near future.

Spindle-cell carcinoma, bre cancer,

Soft tissue sarcoma

Copyright (C) 2021. This is an open-access article distributed under the terms of the Creative Commons Attribution-Non-Commercial 4.0 International License which permits copy and redistribution of the material in any medium or format or adapt, remix, transform, and build upon the material for any purpose, except for commercial purposes.

\section{INTRODUCTION}

Spindle cell carcinoma (SpCC) is an unusual form of squamous cell carcinoma (SCC) where part or all of the tumor resembles a sarcoma. ${ }^{1}$ SpCC can grow in different anatomical locations of the human body such

\footnotetext{
*Address for correspondence:

Amer Abu-Shanab, MD

Address: Office of Scientific Affairs and Research Institute/University/Hospital: King Hussein Cancer Center, Queen Rania St., Amman, 11941, Jordan

Tel: +962789504850

E-mail: amer abushanab23@yahoo.com
}

as the parotid gland, and is most commonly developed from epithelial cells. ${ }^{2}$ Despite the controversy around SpCC, it is regarded as epithelial cancer. ${ }^{3}$ SpCC of the breast is extremely rare, accounting for only $0.1 \%$ of all breast cancers. ${ }^{3,4}$ Owing to the rarity of breast SpCC, a few case studies are available nowadays and proper evidence is scarce. Therefore, determining the suitable diagnostic and therapeutic methods for breast SpCC is challenging. In order to gain more insight into management methods and generate guidelines for breast SpCC, a detailed review of newly diagnosed cases is required. This would be highly beneficial in deciding on the appropriate treatment for patients with newly diagnosed breast SpCC. Here, we present a case 
report of a female patient with right-sided SpCC of the breast resembling inflammatory carcinoma.

\section{CASE PRESENTATION}

A 60-year-old woman, married, nulliparous, nonsmoker with no history of using any contraceptives was studied. Past medical history includes hypertension, which is controlled with once-daily $5 \mathrm{mg}$ tablets of bisoprolol (Bicor). The past surgical history is positive for an open appendectomy 30 years ago. She had no previous exposure to radiation and no family history of breast cancer.

She presented to the clinic with a two-week history of right breast mass which then got ulcerated. Her condition started as she sustained an accidental trauma involving the right side of the chest being hit by an edge of a door. Initially, she had mild pain along with bruising and swelling of the area. Two days later, she noticed an increment in the size of the swelling. After a trial of scrubbing the swelling with a body exfoliating cloth, the mass opened to reveal an ulcer with a foulsmelling yellowish discharge (pus). Consequently, the mass started to shrink in size, unlike the size of the ulcer which started to increase. The patient denies any associated symptoms throughout, such as fever, rigor, or generalized weakness.

Physical examination revealed a right-sided breast ulcerating mass (Figure 1). The mass was hard, immobile, around $7 \times 7 \mathrm{~cm}$ in size, located in the upper outer quadrant. The ulcer was round with a diameter of $3 \mathrm{~cm}$ as shown in Figure 1. The base of the ulcer was yellowish-white in color, and it was surrounded by an elevated firm rim of erythematous thick skin. Moreover, a yellowish foul-smelling pus discharge mixed with bloody was noticed. Thus, the patient was labeled to have an infected ulcerated breast mass. The rest of the examination, including other lesions on the same/contralateral breast, nipple changes/discharge and palpable axillary lymph nodes, was unremarkable.

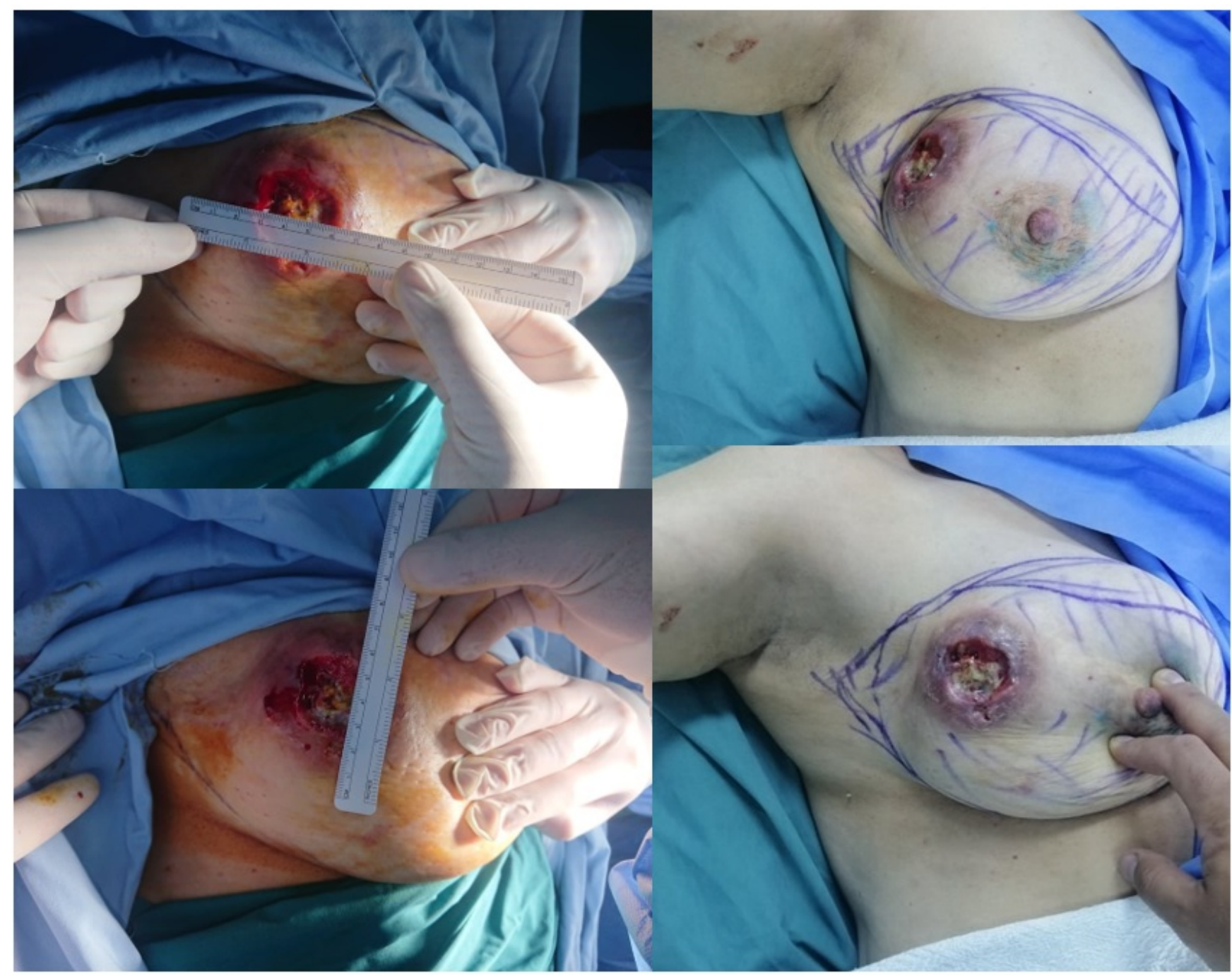

Figure 1. Gross presentation of the breast ulcer 
Given the aforementioned information in addition to the physical examination findings of the patient and her breast, it was essential to rule out inflammatory breast cancer.

\section{Diagnostics}

Arrangements were made for the patient to get further investigations including imaging studies and tru-cut and skin punch biopsies. Her blood tests including full blood count (FBC) and biochemistry showed unremarkable results. On mammography, a large dense lesion measuring approximately $7 \times 7 \mathrm{~cm}$ with focal calcifications was found in the upper outer quadrant of the right breast in addition to thickening of the skin which suggests malignancy. Mammography of the left breast and bilateral axillary lymph nodes was unremarkable. The BI-RADS score for these mammographic findings was $4 .{ }^{5}$ Upon further evaluations with an ultrasound scan of the right axillary area, there were no enlarged lymph nodes. Both tru-cut and skin punch biopsies showed a cellular spindle cell neoplastic lesion. Whole body CT-scan showed a right breast mass $(7 \times 6.5 \mathrm{~cm}$, welldefined with soft tissue density); right renal simple cyst $(2.3 \times 2.3 \mathrm{~cm})$; and fat density of the spleen $(4 \mathrm{~mm})$. CT-scan result showed no evidence of distant metastatic disease.

Therapeutic Intervention

As soon as the results of the biopsies were received from the histopathology department, the patient was admitted to the surgical ward and underwent a right total mastectomy with sentinel lymph node biopsy (SLNB). The operation was smooth and uneventful. Although methylene blue dye was used to recognize the sentinel lymph node, it could not be detected. Therefore, an axillary sampling was performed instead. Tissues removed during the surgery were sent to the histopathology lab which confirmed the diagnosis of spindle cell carcinoma.

\section{Outcome and Follow-up}

Postoperatively, the patient was vitally stable and reported no complications. She was discharged on day one post-operatively. A draining tube was left in place from postoperative day zero and removed on postoperative day two. Thereafter, she had a smooth recovery at home. On the first follow-up visit to the clinic (post-operative day four), she was doing well, and the wound looked healthy and clean. On the second follow-up visit (post-operative day eight), the wound was absolutely fine, and the stitches were removed. Further management with chemo- radiotherapy was offered and discussed with the patient. However, she refused due to the uncertainty of chemo-radiotherapy benefits for this type of tumor.

\section{DISCUSSION}

In the past, the exact definition of SpCC was not clear due to the lack of information. ${ }^{4}$ However, nowadays it is classified by the WHO (World health organization) as a metaplastic type of carcinoma. According to SpCC characteristics and depending on the morphological variety ranging from reactive to malignant lesions, it can be further classified into either benign or malignant tumors. ${ }^{2}$ Additionally, the 5-year survival rate ranges between $28-68 \%{ }^{6}$ The average age of diagnosis of SpCC is 54 years, although our patient was diagnosed at the age of 60 years. $^{3}$

The case series of breast SpCC reported by Khan et al. showed that, macroscopically it could present as an ovoid or round-shaped high-density lesion. ${ }^{4}$ In another study, most patients of SpCC had roundshaped lesions accompanied by decreased elasticity on physical examination. ${ }^{3}$ Mammographically, breast SpCC is usually defined as BI-RADS 4 or BI-RADS 5 with no calcifications. ${ }^{4}$ Our patient had a right-sided ovoid lesion, presented with signs of inflammation and discharge, and the lesion was categorized as BIRADS 4 with local calcifications. These findings suggest that SpCC might have a wider spectrum of mammographic characteristics.

Histomorphologically, SpCC growths are divided into two groups: biphasic - a mixture of carcinomatous and sarcomatous components, or monophasic - largely composed of spindle cells. ${ }^{7} \mathrm{By}$ using immunohistochemistry, specific epithelial and mesenchymal markers can be detected, such as vimentin; cytokeratin (AE1/AE3); cytokeratin 14 (CK14); increased expression of prognostic markers p63 and Ki-67; and reduced expression of prognostic markers ck5/6 and CD10. ${ }^{8}$ In our case, tissue samples from tru-cut and skin punch biopsies were mainly composed of cellular spindle cells in a highly vascular hyalinized stroma and accompanied by an area of acellular hyaline myxoid degeneration (Figure 2). Hence, our patient was diagnosed with a right breast cellular spindle cell neoplastic lesion. Upon immunohistochemical evaluation, the tissue sample was reactive for basal markers CK5/6, p63, vimentin and SMA (Figure 3). It was negative for ER, PR, and HER2/neu (Figure 4), as well as Pan-CK, B-catenin, desmin, and CD34 (Figure 5). 


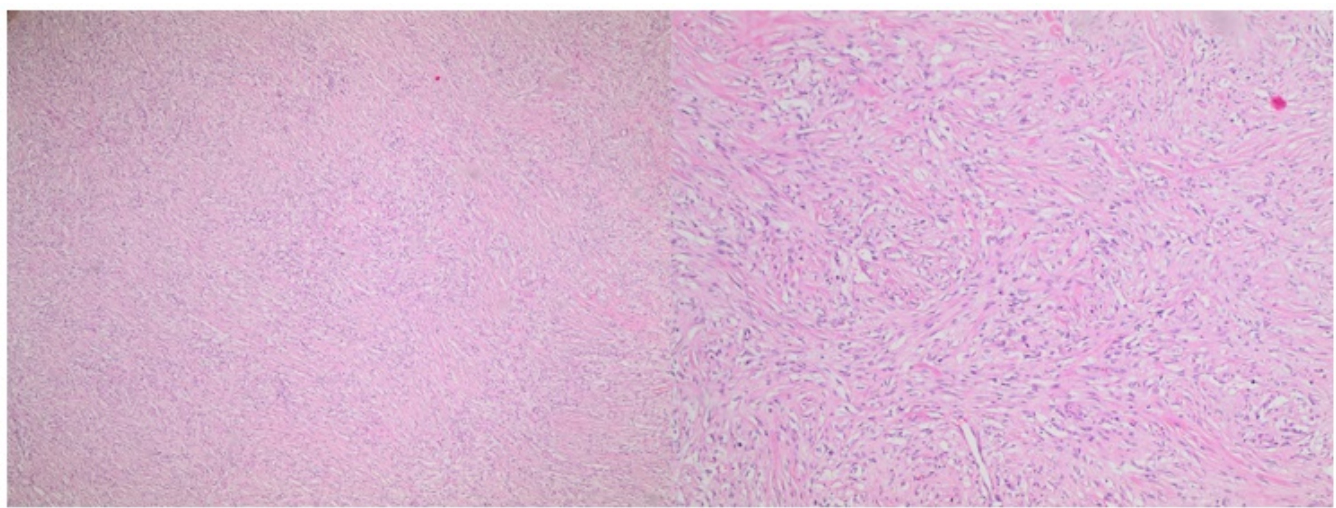

(A) $\times 4$

(B) $\times 10$

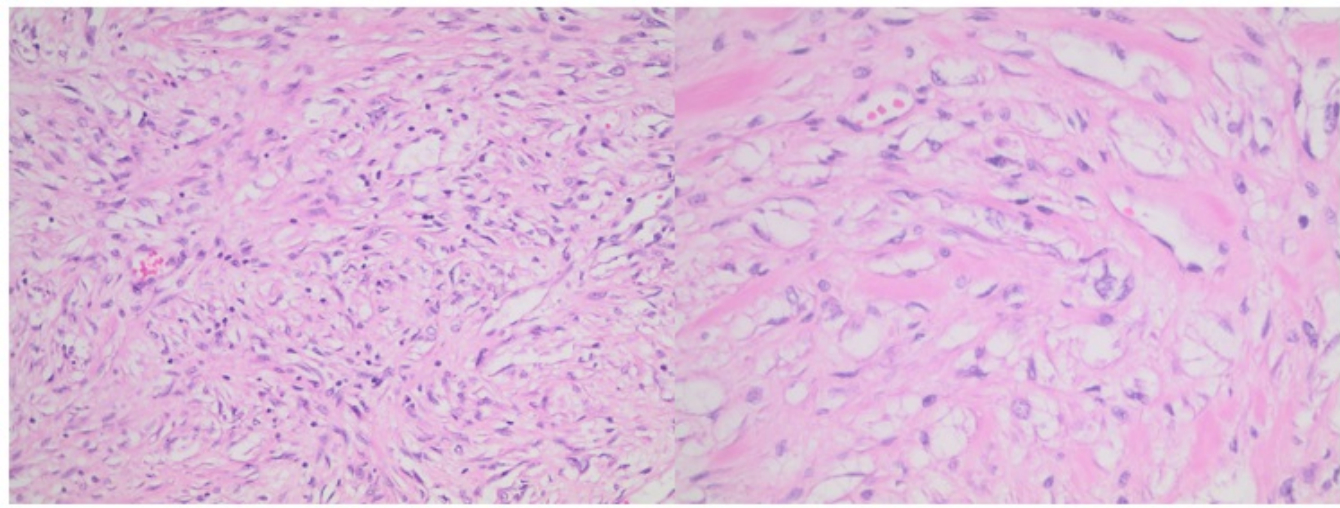

(C) $x 20$

(D) $\times 40$

Figure 2. Hematoxylin and eosin (H\&E) stain. Microscopic examination revealed mild to moderately atypical spindle cells arranged in an interwoven pattern with elongated cytoplasm, mild nuclear pleomorphism was apparent.

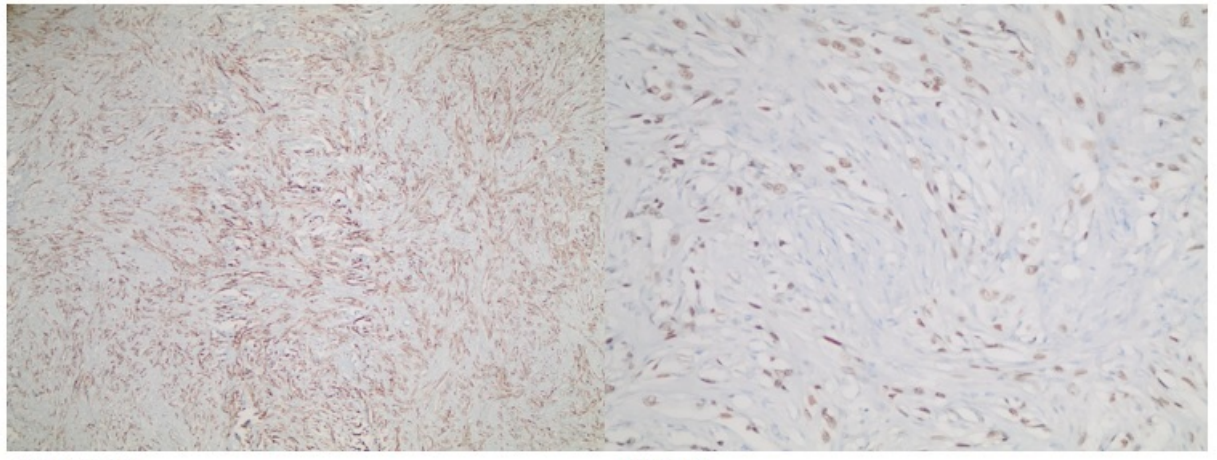

(A) CK 5/6

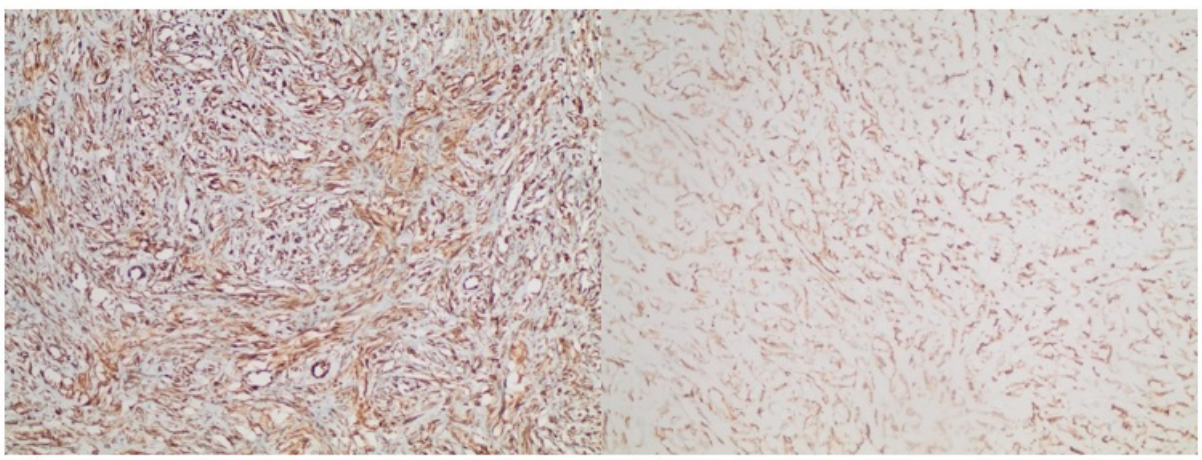

(C) Vimentin

(D) SMA

Figure 3. Immunohistochemistry for CK5/6, P63, Vimentin and SMA highlighting the spindle tumor cells. 


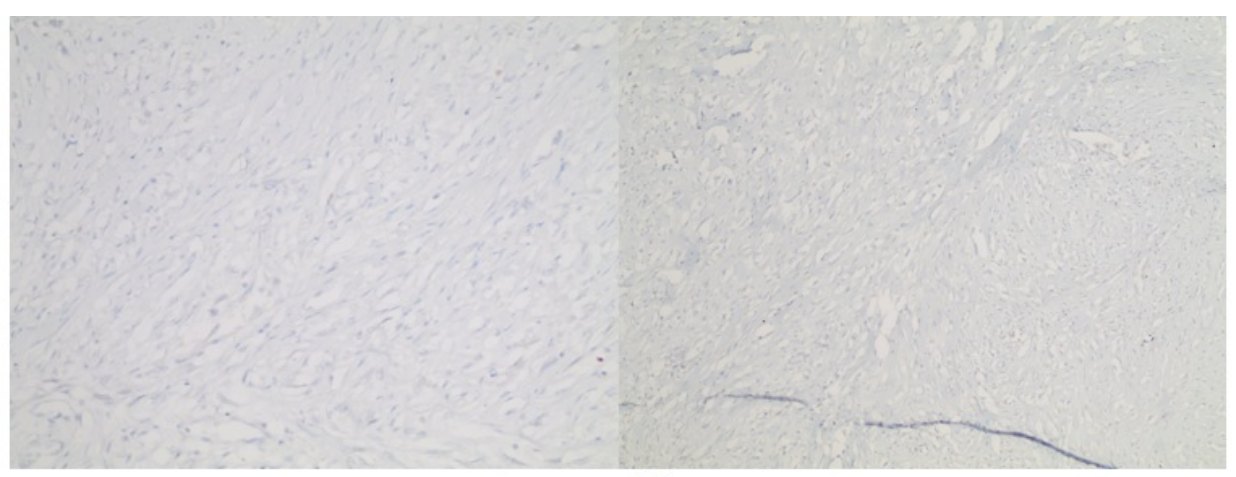

(A) HER2/neu

(B) ER

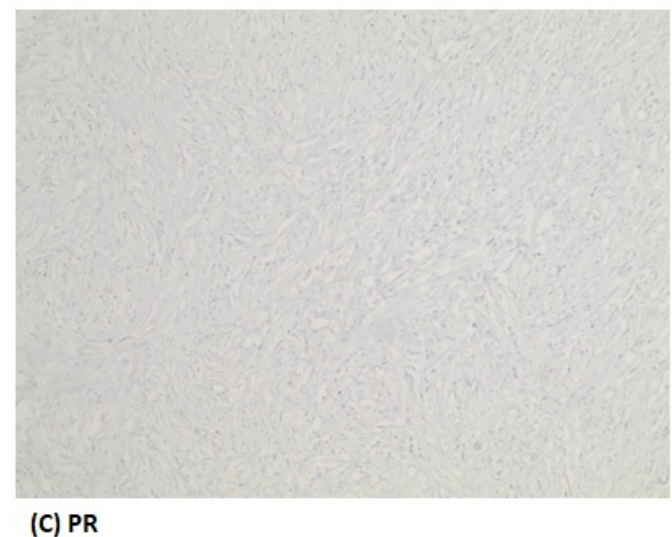

(C) PR

Figure 4. Immunohistochemistry for ER, PR and HER2/neu. The tumor cells are negative for those markers
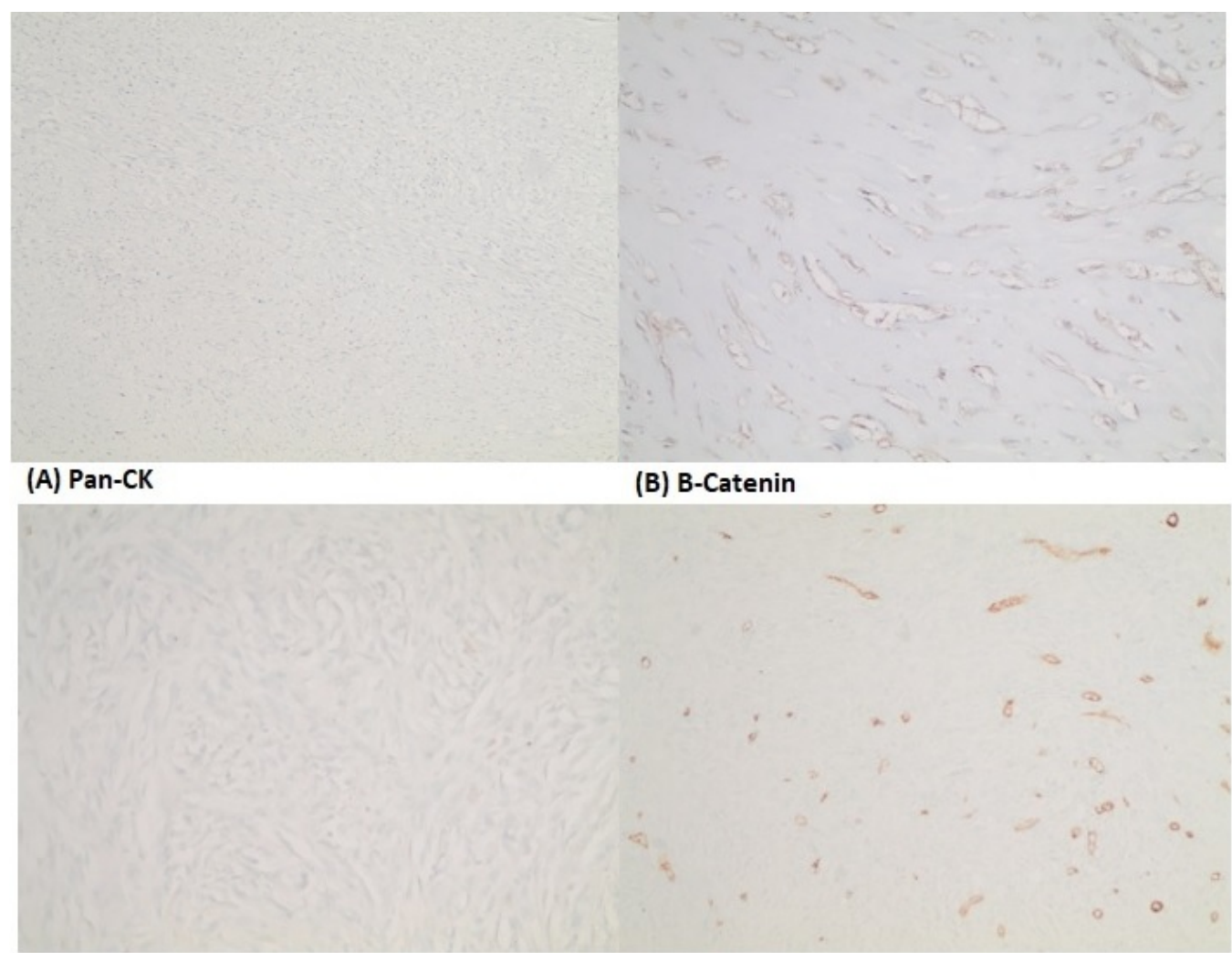

\section{(C) Desmin}

(D) CD34

Figure 5. Immunohistochemistry for Pan-CK, B-Catenin, Desmin and CD34. The tumor cells are negative for all these markers as seen.

It should be noted that SpCC is less likely to metastasize to regional lymph nodes; however, it has a high rate of local recurrence. ${ }^{9}$ In Maemura et al.'s study, ${ }^{3}$ only two out of 12 patients had confirmed 
nodal involvement. Similarly, our patient clinically showed no involvement of axillary lymph nodes. Evidence suggests that tumor size and grade are regarded as more crucial prognostic factors than lymph node involvement. ${ }^{10}$ Carter et al. suggested that tumor size is a strong prognostic factor. ${ }^{10}$ This goes in line with an earlier study by Bauer et al. where the tumor size of more than $2 \mathrm{~cm}$ was associated with higher risks than with smaller tumors. ${ }^{11}$ Results by Song et al. suggest that tumor size of $>5 \mathrm{~cm}, \mathrm{Ki}-67$ of $>14 \%$, and nodal involvement are indicators of poor prognosis. ${ }^{12}$ Not only the size of the tumor but also biomarkers, such as p53 and p63, can potentially indicate poor prognosis. ${ }^{13}$ According to this evidence, our patient was regarded as a high-risk patient due to the big size of the lesion $(7 \mathrm{x} 7 \mathrm{~cm})$ and the reactivity to some markers, mainly p63.

In terms of lymph node dissection, axillary lymph node dissection (ALND) is regarded as a conflicting issue. Podetta et al. in a study on fibromatosis-like spindle-cell metaplastic carcinoma (FLSpCC) found that the need for ALND depends on the type of cancer. ${ }^{14}$ Given the fact that FLSpCC has a higher local recurrence rate than regional lymph node involvement, ALND would be unnecessary. Alternatively, wide local excision or mastectomy with negative margins can be performed in patients with FLSpCC. ${ }^{14}$ Despite the low risk of lymph node involvement, some authors have stated that axillary ultrasound (AUS), fine needle aspiration (FNA), and sentinel lymph node biopsy are quite reasonable for accurate nodal status assessment and treatment. ${ }^{15}$ Our patient had no palpable axillary lymph nodes; hence, AUS and SLNB were performed for exact staging. Intraoperatively, none of the axillary lymphatics was stained by methylene blue. Therefore, we decided to proceed with axillary sampling. Although six axillary lymph nodes were sampled, none of them were found to be carcinogenic in the final pathology report.

Due to the rarity of breast SpCC, the exact treatment plan is not yet established. Historically, mastectomy has been considered the mainstay treatment for patients with big-sized SpCC tumors. ${ }^{11}$ Nevertheless, provided that SpCC does not tend to metastasize to lymph nodes, it is still unclear whether it is mandatory to perform axillary surgery. Mastectomy versus conservative surgery is also a matter of debate. ${ }^{16}$ While gold-standard treatment for SpCC was total mastectomy, ${ }^{11}$ the 10 -year survival rate was higher following total mastectomy than conservative surgery, $82.5 \%$ vs $61.5 \%$, respectively. ${ }^{16}$ Therefore, nowadays, mastectomy is being used for patients with large SpCC tumors. ${ }^{17}$ Moreover, conservative surgery can be performed in patients with early-stage SpCC, while radical mastectomy is highly recommended for late-stage disease, ${ }^{16}$ which highlights the importance of staging in these cases. By taking the aforementioned information into consideration (i.e., the importance of tumor size and stage), the presented case underwent right-sided total mastectomy with sentinel lymph node dissection. She had a big-sized SpCC, and the pathological stage was reported to be pT4N0M0, G2.

According to Moten et al.'s study, ${ }^{16}$ most patients who were diagnosed with SpCC had triple-negative histo-type (i.e., negative ER, PR, and HER2/neu receptor), while only $15 \%$ had $\mathrm{ER}+$ receptor status. The same results were reported in the study conducted by Vranic et al., ${ }^{18}$ where 21 out of 23 patients had triple-negative breast SpCC. Similarly, our patient had triple-negative ER-, PR-, and HER2/neu histotype. Therefore, the major drawback of this histo-type is the lack of hormone receptors (i.e., target cells) and, consequently, hormonal therapy would not be effective. ${ }^{19}$ Neoadjuvant chemotherapy is still an option, mostly for big-sized SpCC tumors, which can help the surgeons to achieve negative resection margins. ${ }^{20}$ However, Chen et al. applied a neoadjuvant chemotherapy setting and their study showed no benefits in terms of tumor shrinkage and downstaging. ${ }^{21}$ Furthermore, chemotherapy for metastatic disease showed a very low response rate. ${ }^{22}$ However, chemotherapy was offered to our patient, but the side effects of the chemotherapy and the low response rate were the reasons behind the patient's refusal to proceed with further treatment after surgical resection.

Nevertheless, in the pioneering study conducted by Zhou et al., apatinib -tyrosine kinase inhibitor (TKI)- was used after unsuccessful treatment of SpCC by using chemotherapy plus cyclophosphamide and epirubicin. ${ }^{23}$ The study showed promising results in terms of nearly complete response to treatment accompanied by tolerable toxicity. These results raise hope as they suggest an effective treatment option for resistant cases. Vranic et al.'s study is considered influential as they elucidated prospective biomarkers for SpCC. Out of 23 patients, 21 had specific mutations, such as PIK3CA, TP53, HRAS, NF1, and PTEN..$^{18}$ This is an evident indicator that personalized medicine is the future of onco-patient treatment, especially for rare types of cancers. We did not perform genetic profiling for specific gene mutations; hence, our patient was planned to be treated as triplenegative breast cancer. This treatment option consists of chemotherapy in combination with taxane and anthracycline in the adjuvant setting. ${ }^{24}$ In case of recurrence, dual therapy (i.e., chemotherapy plus TKIs, such as lapatinib or apatinib) can be incorporated.

The benefits of adding radiation therapy (RT) to surgery is still unclear as the results reported by 
several studies are still controversial. Tseng et al.'s study, ${ }^{25}$ demonstrated improved survival rates in patients who received RT in combination with surgery, while Moten et al. showed worse survival rates with the same combination of treatment. ${ }^{16}$ Consequently, in order to decide on which treatment is the best option, this can potentially be done on a case-by-case basis. This means that personalized medicine in combination with further investigations for each patient is the best approach in the current situation. For our patient, the multidisciplinary team is aiming to decide on a future treatment plan.

\section{CONCLUSION}

Owing to the heterogeneity of SpCC, there is no exact treatment protocol for this type of cancer. Nevertheless, mastectomy or conservative surgery can be performed in certain groups of patients depending on tumor size, stage, and lymph node involvement. Before surgery, AUS and FNA must be

\section{REFERENCES}

1. Bavle RM, Govinda G, Venkataramanaiah PG, Muniswamappa S, Venugopal R. Fallacious Carcinoma- Spindle Cell Variant of Squamous Cell Carcinoma. J Clin Diagn Res. 2016;10(7):Zd05-8.

2. Wei S, Hameed O. Spindle-cell lesions of the breast with emphasis on the differential diagnosis. The Open Breast Cancer Journal. 2010;2(1).

3. Maemura M, Iino Y, Oyama T, Hikino T, Yokoe T, et al. Spindle cell carcinoma of the breast. Jpn J Clin Oncol. 1997;27(1):46-50.

4. Khan HN, Wyld L, Dunne B, Lee AH, Pinder SE, et al. Spindle cell carcinoma of the breast: a case series of a rare histological subtype. Eur J Surg Oncol. 2003;29(7):600-3.

5. Mercado CL. BI-RADS update. Radiol Clin North Am. 2014;52(3):481-7.

6. Okushiba S, Minagawa H, Shimizu M, Ambo Y, Kaji $\mathrm{M}$, et al. A case of spindle cell carcinoma of the breast--long survival achieved by multiple surgical treatment. Breast Cancer. 2001;8(3):238-42.

7. Tse GM, Tan PH, Lui PC, Putti TC. Spindle cell lesions of the breast--the pathologic differential diagnosis. Breast Cancer Res Treat. 2008;109(2):199207.

8. Alaoui M'hamdi H, Abbad F, Rais H, Asmouki H, Soumani A, et al. Rare variant of metaplastic carcinoma of the breast: a case report and review of the literature. J Med Case Rep. 2018;12(1):43.

9. Gobbi H, Simpson JF, Borowsky A, Jensen RA, Page DL. Metaplastic breast tumors with a dominant fibromatosis-like phenotype have a high risk of local recurrence. Cancer. 1999;85(10):2170-82. performed to find out whether ALND is required or not. Chemotherapy is a treatment option in adjuvant and neoadjuvant settings. However, the neoadjuvant setting did not show satisfactory effectiveness in terms of downstaging. A novel drug, such as apatinib (TKI), is a promising option for patients with previously ineffective chemotherapy treatment. Biomarkers are also essential in choosing the appropriate drugs for each patient. However, further research is required to declare which treatment option is most appropriate.

\section{ETHICAL CONSIDERATIONS}

Written informed consent was obtained from the patient herself for publication of this case report and any accompanying images in this journal.

\section{CONFLICT OF INTEREST}

None.

10. Carter MR, Hornick JL, Lester S, Fletcher CD. Spindle cell (sarcomatoid) carcinoma of the breast: a clinicopathologic and immunohistochemical analysis of 29 cases. Am J Surg Pathol. 2006;30(3):300-9.

11. Bauer TW, Rostock RA, Eggleston JC, Baral E. Spindle cell carcinoma of the breast: four cases and review of the literature. Hum Pathol. 1984;15(2):14752.

12. Song $Y$, Liu X, Zhang G, Song H, Ren Y, et al. Unique clinicopathological features of metaplastic breast carcinoma compared with invasive ductal carcinoma and poor prognostic indicators. World J Surg Oncol. 2013;11:129.

13. Zhang Y, Lv F, Yang Y, Qian X, Lang R, et al. Clinicopathological Features and Prognosis of Metaplastic Breast Carcinoma: Experience of a Major Chinese Cancer Center. PLoS One. 2015;10(6):e0131409.

14. Podetta M, D'Ambrosio G, Ferrari A, Sgarella A, Dal Bello B, et al. Low-grade fibromatosis-like spindle cell metaplastic carcinoma: a basal-like tumor with a favorable clinical outcome. Report of two cases. Tumori. 2009;95(2):264-7.

15. Murphy BL, Fazzio RT, Hoskin TL, Glazebrook KN, Keeney MG, et al. Management of the axilla in metaplastic breast carcinoma. Gland Surg. 2018;7(2):200-6.

16. Moten AS, Jayarajan SN, Willis AI. Spindle cell carcinoma of the breast: a comprehensive analysis. Am J Surg. 2016;211(4):716-21.

17. Shah DR, Tseng WH, Martinez SR. Treatment options for metaplastic breast cancer. ISRN Oncol. 2012;2012:706162. 
18. Vranic S, Stafford P, Palazzo J, Skenderi F, Swensen J, et al. Molecular Profiling of the Metaplastic Spindle Cell Carcinoma of the Breast Reveals Potentially Targetable Biomarkers. Clin Breast Cancer. 2020;20(4):326-31.e1.

19. Kitada M, Hayashi S, Matsuda Y, Ishibashi K, Oikawa K, et al. Spindle cell carcinoma of the breast as complex cystic lesion: a case report. Cancer Biol Med. 2014;11(2):130-3.

20. Pandey M, Mathew A, Abraham EK, Rajan B. Primary sarcoma of the breast. J Surg Oncol. 2004;87(3):121-5.

21. Chen IC, Lin CH, Huang CS, Lien HC, Hsu C, et al. Lack of efficacy to systemic chemotherapy for treatment of metaplastic carcinoma of the breast in the modern era. Breast Cancer Res Treat. 2011;130(1):345-51.
22. Schwartz TL, Mogal H, Papageorgiou C, Veerapong J, Hsueh EC. Metaplastic breast cancer: histologic characteristics, prognostic factors and systemic treatment strategies. Exp Hematol Oncol. 2013;2(1):31.

23. Zhou N, Liu C, Hou H, Zhang C, Liu D, et al. Response to apatinib in chemotherapy-failed advanced spindle cell breast carcinoma. Oncotarget. 2016;7(44):72373-9.

24. Wahba HA, El-Hadaad HA. Current approaches in treatment of triple-negative breast cancer. Cancer Biol Med. 2015;12(2):106-16.

25. Tseng WH, Martinez SR. Metaplastic breast cancer: to radiate or not to radiate? Ann Surg Oncol. 2011;18(1):94-103.

\section{How to Cite This Article}

Athamnah MN, Al-Barrak SM, Rabai NA, Natelauri T, Al Azzam H, et al. Spindle Cell Carcinoma of the Breast: A Case Report Representing Diagnostics, Therapeutics, Intervention, and Outcome. Arch Breast Cancer. 2021; 16(4):350-357

Available from: https://www.archbreastcancer.com/index.php/abc/article/view/430 